\title{
Preparation of Surface-Enhanced Raman Scattering Substrates Based on Immobilized Silver-Capped Nanoparticles
}

\author{
C. Caro ${ }^{1},{ }^{1,2,3}$ F. Gámez, ${ }^{1}$ and A. P. Zaderenko ${ }^{1}$ \\ ${ }^{1}$ Departamento de Sistemas Físicos, Químicos y Naturales, Universidad Pablo de Olavide, Crta. de Utrera Km 1, 41013 Sevilla, Spain \\ ${ }^{2}$ REQUIMTE/UCIBIO, Departamento de Química, Faculdade de Ciências e Tecnologia, Universidade Nova de Lisboa, \\ 2829-516 Caparica, Portugal \\ ${ }^{3}$ Centro Andaluz de Nanomedicina y Biotecnología (BIONAND), Calle Severo Ochoa 35, 29590 Málaga, Spain
}

Correspondence should be addressed to C. Caro; cacarsal@gmail.com

Received 21 January 2018; Accepted 19 February 2018; Published 8 April 2018

Academic Editor: Christoph Krafft

Copyright $\odot 2018$ C. Caro et al. This is an open access article distributed under the Creative Commons Attribution License, which permits unrestricted use, distribution, and reproduction in any medium, provided the original work is properly cited.

\begin{abstract}
Novel results concerning surface-enhanced Raman scattering mediated by thiol-immobilized capped silver nanoparticles attached to a silicon $\mathrm{Si}(100)$ substrate are presented. The attachment of the nanoparticles is achieved by chemically modifying the surface of $\mathrm{Si}(100)$ in order to provide sulfhydryl groups covalently linked to the substrate and then covering these surfaces with bare and polymer-capped silver nanoparticles. The modified silicon substrate, the nanoparticles, and the sensors are characterized by means of infrared and UV-vis spectroscopies and electronic microscopies. The surface-enhanced Raman scattering intensity of the new films based on polymer-capped nanoparticles is compared with that obtained with silver bare nanoparticles using rhodamine $6 \mathrm{G}$ as a common chromophore. These results open a new route to the design of reversible and spot-to-spot reproducible surface-enhanced Raman scattering-based sensors supported by silver nanoparticles.
\end{abstract}

\section{Introduction}

The fast pace development of nanotechnology has afforded new avenues towards the design of new materials and devices in chemical sensing $[1,2]$, nanoelectronics $[3,4]$, or optics [5-8]. At this length scale, size matters and the importance of one atom in electronical, chemical, and optical properties ranging in-between atomic and bulk ones is currently a hot topic [9]. The strong impact provoked by size effects in these features comes mainly from their characteristic surface/volume ratio [10], quantum confinement effect [11, 12], and quantum electrodynamic interactions [13]. To our purposes, the most important quantum size effect is the surface plasmon resonance (SPR) providing light absorption in the visible range by the red shift of the Drude frequency (in the UV range for metals) to the optical region provoked by the modification of the selection rules for polarization induced by the surface electrons. In fact, a plasmon is nothing but a quantized in-phase collective oscillation of polarized "free" conduction electrons confined within a metal/dielectric boundary $[14,15]$. The optical properties of plasmonic nanoparticles can hardly be explained employing traditional theories (as those based in the dipole approximation [16]), but a size dependence is foreseen (and experimentally demonstrated) as a consequence of the differential contributions of the interband transitions (due to $d$ electrons) and Drude parts of the dielectric function, which causes that surficial scattering exceeds that one coming from the bulk [17]. Also, different nanoparticle functionalizations cause changes in the near-surface refractive index $n$ that result in a shift of the absorption maximum [18]. Although these facts can be used to tune the plasmon absorption for being used in surface plasmon resonance (SPR) spectroscopy, one of the most important applications concerning this effect is surfaceenhanced Raman scattering (SERS) [19-23], which aims at reaching ultrasensitive detection, from simple chemical pollutants to biomolecules and immunoassays [24-26] with up to single-molecule sensitivity $[27,28]$. The reason behind this 
huge increment of sensitivity with respect to usual resonant Raman experiments are the increase of the molecular Raman cross section by several orders of magnitude to even $10^{14}$ for some molecule-surface combinations [29].

Two nonexcluding mechanisms have been proposed for the explanation of the SERS effect: (1) the electromagnetic enhancement (EM) and (2) the chemical effect (CE) [30, 31]. In the EM mechanism, the local surface interaction between the incident and local-enhanced scattered electric fields with the transition moment of the adsorbed molecules modifies the Raman transition moment $[32,33]$. Hence, the metallic surface needs to be rough/fractal and highly heterogeneous at the nanoscale, which favors the formation of geometrical gaps between nanoparticles of a few nanometers size causing such a huge enhancement of the electromagnetic fields. The morphology of the particles and the distribution of the nanowells or "hot spots" [34,35] are the main responsible factors for SERS effect through the local field EM mechanism [36]. In the CE mechanism, the mixing of orbitals of molecular and metallic character leads to a charge transfer process that diminishes the energy for the resonant molecular Raman with respect to the energy of the free molecule [28]. Although the EM is of leading order and the CE provides additional enhancement, in order for either or both of these mechanisms (among others) to play a role, from a practical viewpoint, one needs the metal substrate to have certain characteristics especially as far as its nanoparticle is concerned to improve the plasmonic configurations that result in the optimum interparticle interaction for SERS. Hence, the upgrade of metal films with controlled nanostructure and composition is of pivotal importance and is currently intensely investigated $[6,7,37]$. There is a variety of methods for the fabrication of SERS substrates, such as pulsed laser deposition (PLD) [38], metal evaporation [39, 40], chemical or electrochemical etching or deposition on smooth surfaces [41-44], and deposition or assembly of metal nanoparticles from solution (dried droplet (DD) method) [45, 46]. However, for a given excitation wavelength, SERS effect is nearly absent in isolated particles, while the coupling of transition dipoles in aggregates enhances relatively this effect in the nanoscopic scale [47]. Hence, the addition of salt to induce the formation of aggregates offers a robust and simple DDbased route for the design of SERS-based sensor [1, 48, 49] by the price of a certain degree of lack in the reproducibility and stability because of the heterogeneous hot spot distribution. Within the procedure outlined in this paper, the essential aggregation step in silver bare nanoparticles becomes unnecessary to obtain a comparable SERS effect with high batch-to-batch reproducibility. In summary, in this work, we report the fabrication and characterization of two new SERS active films in which polymer-capped metal nanoparticles are attached to the substrate [50] by means of thiol groups that are covalently bonded to the surface of the Si support. The in-depth characterization of the films employing electronic microscopies reveals that this chemical procedure leads to $\mathrm{Si}(100)$ substrates that are covered with single or small fractal-like clusters (a few microns size) of silver nanoparticles. SERS activity has been proven by adsorbing rhodamine $6 \mathrm{G}$ (R6G) as a model compound and recording the Raman spectra on the sensors with suitable geometry for SERS effect. Differences in the fitness of the films for this analytical aim are also discussed in terms of the plasmonic and surface properties of the devices. In all cases, adsorptive anchoring of the analyte to the nanoparticles is reversible, making all of them candidates to be used as chemical sensors.

So this paper is outlined as follows: the description of the materials and methods is shortly reviewed in Section 2. Results and discussion are summarized in Section 3. The main conclusions extracted in the work are finally presented in Section 4.

\section{Materials and Methods}

2.1. Chemicals. All the chemicals are of reagent grade and have been used without further purification: hydroxylamine hydrochloride $\mathrm{NH}_{2} \mathrm{OH} \cdot \mathrm{HCl}$, tetraethylrhodamine hydrochloride (rhodamine 6G), mercaptoacetic acid (MAA), $\mathrm{N}$-(3-dimethylaminopropyl)- $\mathrm{N}$-ethylcarbodiimide (EDC), polyvinylpyrrolidone $\left(\mathrm{C}_{6} \mathrm{H}_{9} \mathrm{NO}\right)_{n}$ with average molecular weight 10000 (PVP10000), and polyvinylpyrrolidone with average molecular weight 29000 (PVP29000) are supplied by Sigma-Aldrich. Potassium nitrate, (3-aminopropyl)trimethoxysilane, $N$-hydroxysuccinimide (NHS), and 2(N-morpholino)ethanesulfonic acid 1-hydrated (MES) are supplied by PanReac. Water was purified using a Milli-Q $(18.2 \mathrm{M} \Omega \cdot \mathrm{cm})$ reagent grade water system from Millipore. As substrates for immobilization, we use high-purity and high-resistivity silicon (100) wafers from Topsil polished on both sides.

2.2. Instrumentation. Transmission electron microscopy (TEM) images were taken in a Philips CM200 microscope operated at $200 \mathrm{kV}$ with a resolution of $2.8 \AA$. The samples were prepared by drying an aqueous solution of nanoparticles on a carbon-coated copper grid.

Scanning electron microscopy (SEM) images were taken with a Hitachi S5200 high-resolution ( $5 \AA$ ) microscope operating at $30 \mathrm{kV}$. Aqueous samples are dried in a carbon-coated copper tape.

The UV-vis spectra were recorded with an Ocean Optics spectrometer equipped with a HR4000 detector with a quartz tray with a light path of $1 \mathrm{~cm}$.

Raman spectra are recorded in a Bruker Senterra confocal Raman microscope equipped with a laser of $785 \mathrm{~nm}$ and a DU420A-OE-152 detector. The 50x objective is used for all the measurements. The slit aperture is fixed to $50 \mu \mathrm{m}$, and the integration time is $100 \mathrm{~s}$ with a laser power of $10 \mathrm{~mW}$. All the spectra are recorded with a resolution of $3 \mathrm{~cm}^{-1}$.

Fourier transform infrared (FTIR) spectra of the solid nanoparticles are recorded in a IFS 66/S Bruker spectrometer with a DTGS detector in the nominal range of 370$7500 \mathrm{~cm}^{-1}$ and resolution of $1 \mathrm{~cm}^{-1}$ in a $\mathrm{KBr}$ pellet (with a nanoparticle: $\mathrm{KBr}$ relation of $1: 30$ in weight).

Centrifugation of nanoparticle solutions is performed in a refrigerated centrifuge Ortoalresa Digicen 20R with a rotor length of $101 \mathrm{~mm}$. 
2.3. Synthesis of Silver Nanoparticles. The synthesis of $\mathrm{AgNO}_{3}$ with $\mathrm{NH}_{2} \mathrm{OH} \cdot \mathrm{HCl}$ as reducing agent has been performed following the method of Leopold and Lendl [51] without further modifications. Briefly, a solution of hydroxylamine hydrochloride and $\mathrm{NaOH}$ with alkaline $\mathrm{pH}(\sim 10)$ is used as reducing agent of the silver salt. The brownish solution so obtained is centrifuged $(3500 \mathrm{rpm}$, $15 \mathrm{~min}$ ) in order to obtain a narrower size distribution. The final solution turns into a yellowish color whose characterization will be described below. This solution will be labeled as $\mathrm{Ag}(\mathrm{H})$ hereinafter.

Two subsequent functionalizations are then achieved with polyvinylpyrrolidone (PVP) of different average molecular weights (10000 and 29000). These heavy polymers provide amphiphilic character, with a hydrophobic polyvinyl chain and a hydrophilic pyrrolidonic ended group, and are employed for tuning both the nanoparticles shape and the plasmon resonances of the Ag nanoparticles towards the laser wavelength. In the cases of the $\mathrm{AgNO}_{3}$ with PVP10000 and PVP29000 syntheses, the method of Washio et al. [52] has been followed with minor changes in the heating temperature, time of reduction, and silver/ PVP proportion. For the PVP10000 case, $8 \mathrm{~mL}$ of an aqueous solution of PVP $23 \mathrm{mM}$ is kept in a glass flask submerged in a bath oil at $85^{\circ} \mathrm{C}$ under magnetic agitation. Once PVP is properly dissolved, $3 \mathrm{~mL}$ of a $\mathrm{AgNO}_{3}$ solution (Ag/PVP ratio of 30 ) is added quickly and let the reaction occur for six hours. Then, the sample was centrifuged during 30 minutes at $8000 \mathrm{rpm}$. Solid was washed twice with Milli-Q water and centrifuged for another 30 minutes at $8000 \mathrm{rpm}$. After this process, a dun color solution is finally obtained. When the functionalization with PVP29000 is achieved, the procedure to be followed is quite similar, and only the temperature of the oil bath is changed to a lower temperature $\left(65^{\circ} \mathrm{C}\right)$. In this synthesis, a green solution is obtained for the final nanoparticle dispersion. These nanoparticles will be tagged as Ag@PVP10000 and Ag@PVP29000, respectively, along the text.

\subsection{Substrate Preparation for Surface-Enhanced Raman} Spectroscopy. Films for SERS are produced following the method in [46]. Briefly, the procedure starts with oxidation of the $\mathrm{Si}(100)$ surfaces in a freshly prepared piranha solution $\left(\mathrm{H}_{2} \mathrm{O}_{2}: \mathrm{H}_{2} \mathrm{SO}_{4}, 1: 3(v / v)\right)$ for one hour. This oxidation process results in the formation of a silanol-covered $\mathrm{SiO}_{2}$ layer. Subsequently, the film is reacted with (3-aminopropyl)trimethoxysilane for two hours in order to form an amineterminated monolayer that is then immersed for $2 \mathrm{~h}$ in a MES buffer solution containing MAA that has been activated with EDC + NHS. The carboxylic group of the MAA couples with the amino layer with the corresponding formation of the amide bond providing substrates covered with $\mathrm{SH}$ groups. Subsequent immersion during $1 \mathrm{~h}$ in a suspension of silver nanoparticles (aggregated in $\mathrm{KNO}_{3} 0.1 \mathrm{M}$ in the case of $\mathrm{Ag}(\mathrm{H})$ suspension) covers the substrates with a layer of nanoparticles that are strongly attached to the sulfhydryl groups. The films are washed thoroughly during $1 \mathrm{~h}$ in Milli-Q water after each of these steps. The samples for Raman spectroscopy are prepared by dipping the nanoparticle layers in aqueous solutions or R6G of several concentrations and drying with nitrogen at room temperature.

\section{Results and Discussion}

3.1. Treated Substrate Characterization. The characterization of the silicon substrates has already been described in [46] and will be sketchily reproduced in the supplementary material (available here) for the sake of clarity and completeness of the text.

3.2. Characterization of Silver Nanoparticles. The reduction of $\mathrm{AgNO}_{3}$ in aqueous solution with $\mathrm{NH}_{2} \mathrm{OH} \cdot \mathrm{HCl}$ yields chloride-protected $\mathrm{Ag}$ polyhedral nanoparticles with typical sizes between 20 and $50 \mathrm{~nm}$ as determined by TEM (Figure 1(a)). The aqueous colloidal suspensions of these particles have a plasmon band centered at $406 \mathrm{~nm}$ in the UV-vis absorbance spectrum (see Figure 2). The absorption of valence electrons to the conduction band occurring at a shorter wavelength is not shown in the spectrum. The spectrum of the aggregates (amplified by a factor of two), developed with the help of the addition of a $\mathrm{KNO}_{3}$ solution, is also presented in Figure 2 for comparison. It can be observed that the induced aggregates feature a UV-vis spectrum strongly shifted to the red. This displacement of the absorption maximum provoked by the curling up of the nanoparticles makes them much more adequate given the closeness of the maximum of the band to the excitation laser wavelength. A detailed study of how the stability of this nanoparticle suspension can be tuned by a sequential addition of $\mathrm{KNO}_{3}$ will be given here in view of Figure 3. According to the wellknown Derjaguin and Landau and Verwey and Overbeek (DLVO) theory, the increment of the ionic strength included in the inverse Debye length $\kappa$ redounds in a screening effect of the surface charge that diminishes the energetic barrier $E_{\mathrm{a}}$ between the repulsive (van der Waals force) and the attractive (electroosmotic) interactions. The global effect is the aggregation or precipitation of the nanoparticles as can be observed in the energetic diagram of Figure 3 where the contributions to the total potential energy $\phi(r)$ as a function of the interparticle distance are plotted. The evolution of the absorption spectrum upon the consecutive addition of the potassium salt is presented with some representative samples. As long as the salt concentration increases, the yellowish emission typical of this suspension decreases in favor of the blue one belonging to nanoparticle aggregates. It can also be mentioned that the flattening emerging from the red part of the spectrum is a consequence of a heterogeneous size distribution of the new-formed aggregates. As mentioned in the previous section, this fact is used to increase the number of aggregates after immobilization on the silicon surface since, although both single nanoparticles and small clusters exist, only clusters contribute to SERS effect in the films constructed with these nanoparticles under the experimental conditions because the plasmon resonance of isolated particles is far from the laser wavelength. $\mathrm{AgNO}_{3}$ reduced with PVP10000 forms a narrow-size distribution of polyhedral structures of $\mathrm{Ag}$ nanoparticles, with a size average between 80 and $90 \mathrm{~nm}$ (Figure 1(b)). The maximum of the plasmon 


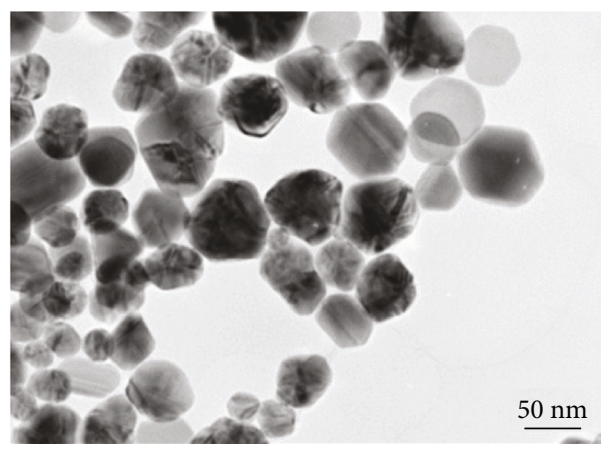

(a)

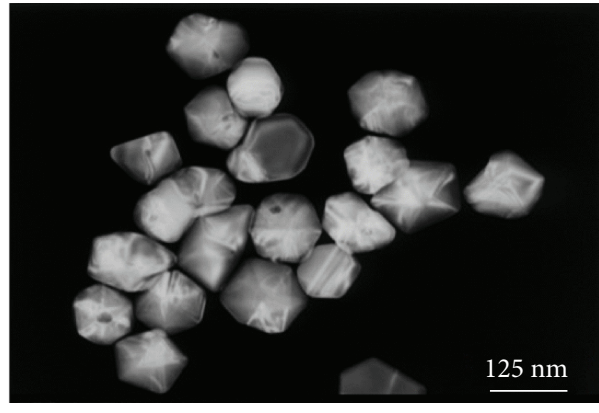

(b)

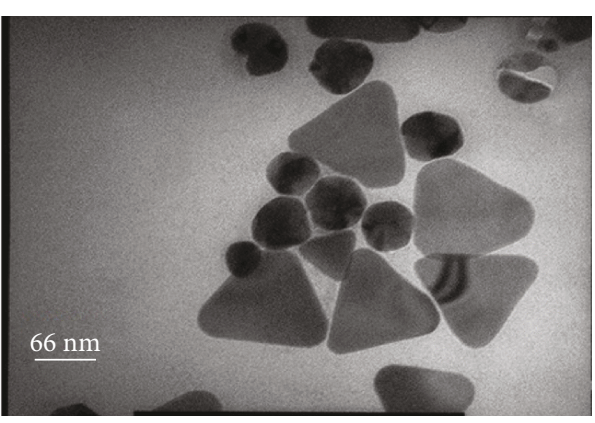

(c)

FIGURE 1: Transmission electron microscopy images of the synthesized nanoparticles. After the synthesis, a droplet of the colloid has been dried out on a copper grid in all cases. (a) $\mathrm{AgNO}_{3}$ reduced with $\mathrm{NH}_{2} \mathrm{OH} \cdot \mathrm{HCl}$. (b) $\mathrm{AgNO}_{3}$ reduced with polyvinylpyrrolidone-10000. (c) $\mathrm{AgNO}_{3}$ reduced with polyvinylpyrrolidone-29000.

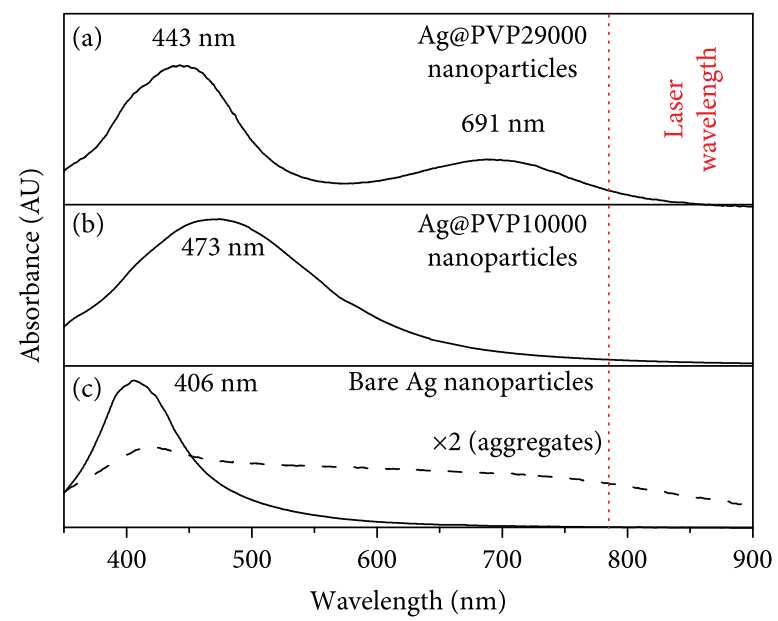

FIGURE 2: UV-vis spectrum of the synthesized nanoparticles. (a) $\mathrm{AgNO}_{3}$ reduced with $\mathrm{NH}_{2} \mathrm{OH} \cdot \mathrm{HCl}$ and their aggregates (upon the addition of $100 \mu \mathrm{L}$ of $\mathrm{KNO}_{3}$ ), (b) $\mathrm{AgNO}_{3}$ reduced with polyvinylpyrrolidone-10000, and (c) $\mathrm{AgNO}_{3}$ reduced with polyvinylpyrrolidone- 29000 .

band of the colloidal suspensions is located at $473 \mathrm{~nm}$ in the UV-vis spectrum (see Figure 2), although the band is the broadest with a tail extending to the near infrared among the nanoparticles considered in this work because of the coupling of the plasmonic bands arising from the different irregular facets of the nanoparticles. In the reduction process with PVP29000, a mixture of nanotriangular plates, in the $115-130 \mathrm{~nm}$ range, and polyhedral nanoparticles with size distribution centered at $60-65 \mathrm{~nm}$ is obtained (see Figure 1(c)). These nanoparticles in aqueous colloidal suspensions will then present three plasmonic bands as observed in Figure 2. One of them will be the one associated with the polyhedral nanoparticles centered at a similar position at the $\operatorname{Ag}(\mathrm{H})$ solution (seen as a shoulder at $\sim 390 \mathrm{~nm}$ in this case). The other two bands, with centers located at $443 \mathrm{~nm}$ and $691 \mathrm{~nm}$, correspond to the transversal and longitudinal plasmon excitations of the nanoparticles with triangularshape geometry, respectively. None of the Ag@PVP-protected nanoparticles aggregates upon the addition of salt, because of the high stability provided by the steric interactions between the high molecular weight coating. Hence, upon evaporation of the solvent, the only modification caused by the interparticle interaction is a broadening of the plasmon bands (not shown). The longitudinal plasmon absorption of the nanotriangles is quite close to the excitation laser radiation, which, a priori, will favor the suitability of these films as SERS-based sensors.

Figure 4 shows the SEM images of some representative aggregates of the nanoparticle covering the substrates. These images allow us to evaluate the surface area, coverage of nanoparticles, and aggregate size of these SERS substrates. Panel (a) shows the $\operatorname{Ag}(\mathrm{H})$ case, with a few hundred $\mu \mathrm{m}^{2}$ area covered with fractal nanoparticle aggregates. Areas with a lesser degree of coverage ranging from single particle areas to small clusters of several $\mu \mathrm{m}^{2}$ are also presented. In the high-resolution image shown in the inset, it can be observed that the morphology of these clusters is comprised of numerous sites separated a few Angstrom that are precisely the 


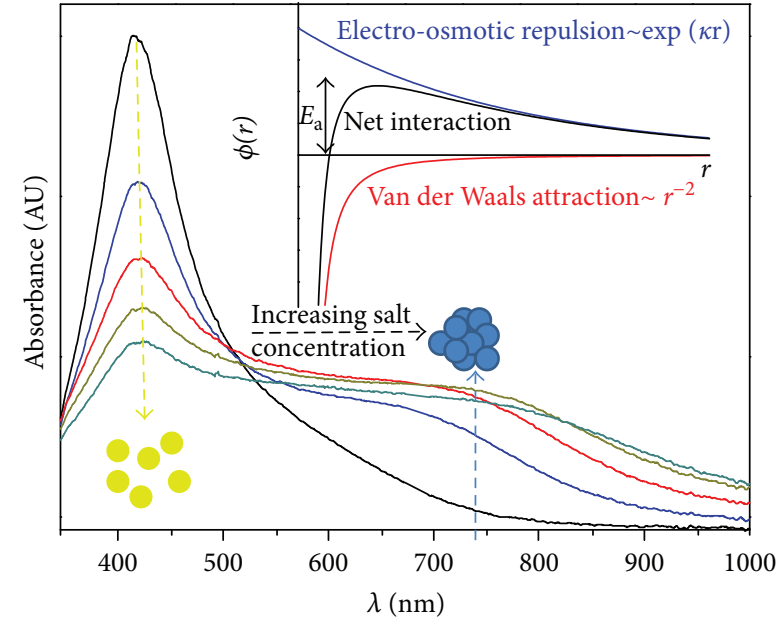

Figure 3: Effect of the ionic strength on the stability of hydroxylamine-reduced silver nanoparticles. The increment of the salt concentration enhances the formation of aggregates absorbing in the red part of the spectrum. The inset shows the energetic balance of the phenomenon according to the Derjaguin and Landau and Verwey and Overbeek (DLVO) theory as explained in the text.

definition of a Raman active "hot spots." Both flat and, to a much lesser extent, spherical-like and thicker aggregates are presented. Flat structures have presumably grown by induced aggregation while the thick ones have been preformed in solution. Since the Ag@PVP nanoparticles are much more stable than the $\operatorname{Ag}(\mathrm{H})$ and, hence, they hardly aggregate together with the fact that the coating prevents the direct chemical interaction with the $\mathrm{SH}$ groups covering the surface, the size and degree of coverage of the aggregates on the sensors built with these nanoparticles diminish with the massiveness of the polymer. In these cases, aggregates typically fill an area of a few hundredths of $\mu \mathrm{m}^{2}$ for PVP10000 and the coverage is even less for PVP29000 (about $100 \mu \mathrm{m}^{2}$ ). Although they also constitute hot spots as shown in the inset of panels (b) and (c), those that belong to PVP10000 are the biggest among the aggregates considered $(\sim 100-500 \mathrm{~nm})$. Consequently, the leading responsible facts of the final performance of each sensor are the detailed balance between plasmon absorption bands and covering effects together with the subtle effect of the analyte-coating molecular interactions.

3.3. SERS Effect in TICSNP Films. Before recording SERS spectra, contributions of R6G fluorescence signals and large surface area effects of the aggregates have been ruled out (see supplementary material). For the SERS experiments, two deposition methods have been followed with nearly identical results: (a) $100 \mu \mathrm{L}$ of R6G at different concentrations (from $10^{-7}$ to $10^{-3} \mathrm{M}$ ) is spread on a target of $0.8 \mathrm{~cm}^{2}$ and (b) incubation of the films in several solutions of R6G at different concentrations $\left(10^{-3} \mathrm{M}\right.$ to $\left.10^{-7} \mathrm{M}\right)$ for $1 \mathrm{~h}$ and then drying them quickly under a stream of pure nitrogen. The silicon slits are observed in the optical Raman microscope. It is also noticeable that these aggregates do not

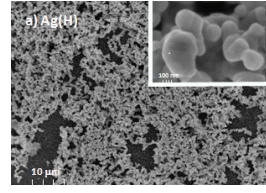

(a)

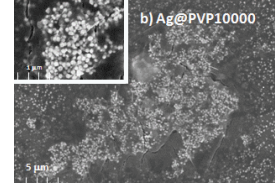

(b)

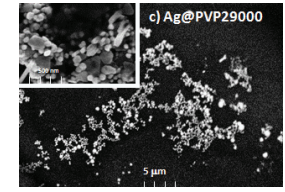

(c)
FIgURE 4: Scanning electron microscopy images of the synthesized nanoparticles. After the synthesis, a droplet of the colloid has been dried out on a copper grid in all cases. (a) $\mathrm{AgNO}_{3}$ reduced with $\mathrm{NH}_{2} \mathrm{OH} \cdot \mathrm{HCl}$. (b) $\mathrm{AgNO}_{3}$ reduced with polyvinylpyrrolidone10000. (c) AgNO3 reduced with polyvinylpyrrolidone-29000.

change their morphology apparently after these processes. Main Raman shift bands (labeled in Figure 5 from A to F in increasing wavenumber order) correspond to xanthene $\mathrm{C}-\mathrm{C}-\mathrm{C}$ ring in-plane bending $\left(611 \mathrm{~cm}^{-1}, \mathrm{~A}\right)$, xanthene $\mathrm{C}-\mathrm{H}$ bending out of plane $\left(770 \mathrm{~cm}^{-1}, \mathrm{~B}\right)$, convolution of ethylamine C-C stretching and C-H bending $\left(1190 \mathrm{~cm}^{-1}, \mathrm{C}\right)$, xanthene $\mathrm{C}-\mathrm{H}$ bending $\left(1310 \mathrm{~cm}^{-1}, \mathrm{D}\right)$, convolution of xanthene $\mathrm{C}-\mathrm{C}$ stretching and xanthene-phenyl C-C stretching $\left(1354 \mathrm{~cm}^{-1}, \mathrm{E}\right)$, and xanthene C-C stretching $\left(1504 \mathrm{~cm}^{-1}, \mathrm{~F}\right)$ [53]. The Raman spectrum of the zones that are covered with single particles or small clusters does not yield appreciable signal from R6G, and only the Raman peak of Si centered at $522 \mathrm{~cm}^{-1}$ is presented. It is well known that the intensity of the Raman signal measured for a given aggregate depends on the amount of SERS active spots in the focused volume. Hence, the difference in Raman intensity obtained when recording the spectrum from a flat and a thick aggregate is ascribed to the smaller number of hot spots contained in the first case. For this reason, it is not feasible to relate the intensity of the Raman signal with concentration by using the recorded spectra. Such a drawback can be overcome by rescaling all the spectra so that they all have the same area under the band centered at $230 \mathrm{~cm}^{-1}$, the $\mathrm{Ag}-\mathrm{Cl}$ stretching. Since the cleaning process removes contributions to the Ag-Cl band that may come from precipitated salt [54, 55], only the contribution of the adsorption of $\mathrm{Cl}^{-}$on $\mathrm{Ag}$ nanoparticles is measured. Hence, such area must be strictly proportional to the number of nanoparticles in the focal region. This rescaling for the average spectra of five measurements is shown in Figure 5 for the different sensors constructed in this work when the $10^{-3} \mathrm{M}$ solution of R6G is used. For Ag@PVP29000-based films, the high molecular weight coating efficiently screens the dipoles coupling, enhancing the reproducibility among different spectra to the order of $30 \%$ as can be seen in Figure 6, regardless of the structure of the probe area. In contrast, the sensitivity of the sensors containing $\operatorname{Ag}(\mathrm{H})$ nanoparticles is highly dependent on the probe area, being necessary to search for an appropriate aggregate in order to obtain a reliable SERS spectrum. One should notice that this procedure overcomes some spectrum artifacts. For example, after normalization, on Ag@PVP10000, the SERS signal of R6G is very weak, although the $\mathrm{Ag}-\mathrm{Cl}$ stretching band remains modest. This would reflect how far the SPR is from the excitation light, a fact that meets with failure in the acquiring of a proper SERS spectrum. 

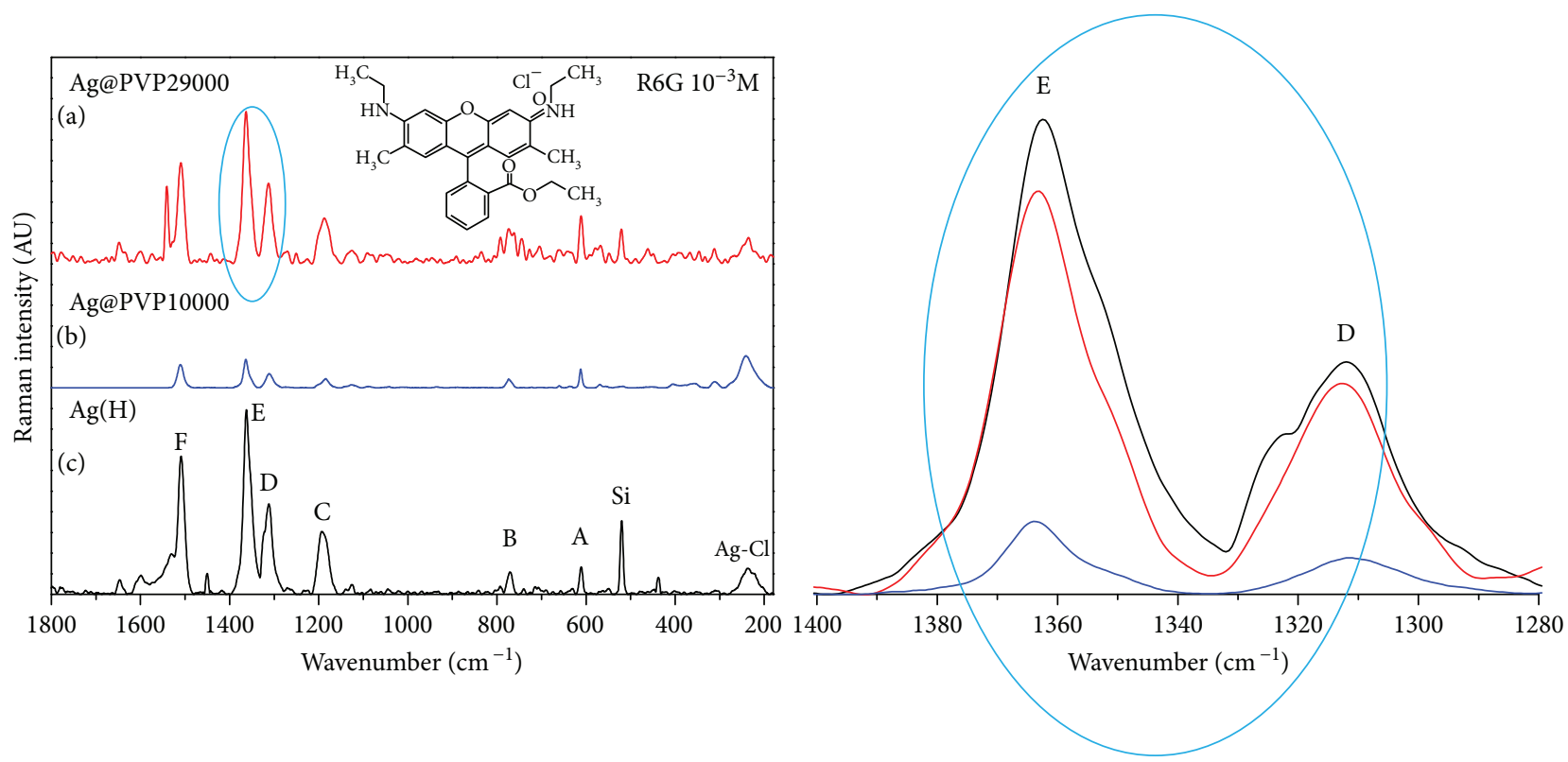

(d)

FIGURE 5: Surface-enhanced Raman scattering spectra of a rhodamine $6 \mathrm{G} 10^{-3} \mathrm{M}$ solution recorded at $785 \mathrm{~nm}$ on sensors composed by Ag@polyvinylpyrrolidone-29000 (a), Ag@polyvinylpyrrolidone-10000 (b), and $\operatorname{Ag}(\mathrm{H})$ (c). Main Raman bands of rhodamine 6G are labeled from A to F (see text for details and assignation to molecular vibrations). (d) A zoom of two of the most prominent bands is shown for quantitative comparison of their Raman intensity normalized with the area of the Ag-Cl stretching band.

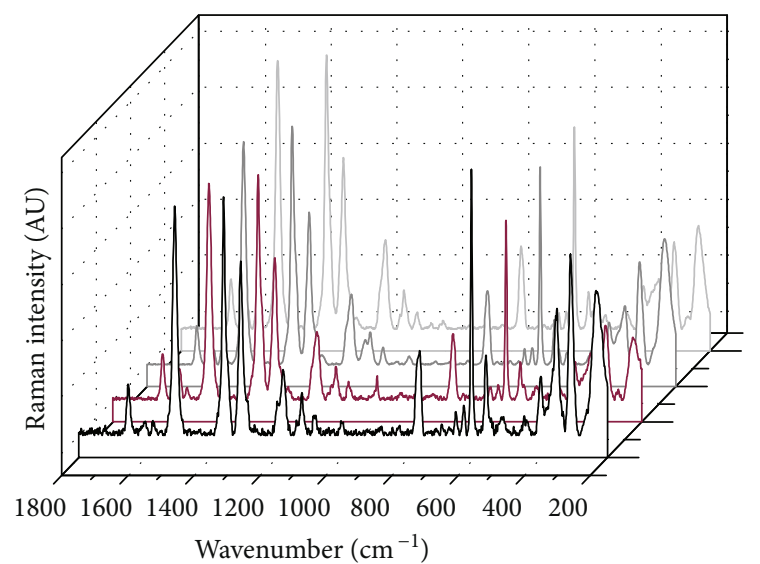

FiguRE 6: Four surface-enhanced Raman scattering spectra recorded on the Ag@polyvinylpyrrolidone-29000-based sensor after incubation with rhodamine $6 \mathrm{G} 10^{-3} \mathrm{M}$ for $1 \mathrm{~h}$. Measurements have been taken at random position with a separation of at least $0.2 \mathrm{~cm}$. The reproducibility among different spectra is within the order of $30 \%$.

Moreover, since many variables are associated with the adsorption mechanism and reproducibility of the scattering volume [56], masking a proper determination of the enhancement factor, the molar sensibility will be presented in the following (i.e., the estimated number of moles probed by the laser spot). These molecular quantities can be easily derived from the laser spot and the area covered with the R6G solution. Thus, the SERS spectrum is observed for an amount as small as $10 \mathrm{zmol}$ in the most unfavorable case $\left(10^{-7} \mathrm{M}\right)$ for $\operatorname{Ag}(\mathrm{H})$ and PVP29000 sensors, but the
Ag@PVP10000-based sensor only reaches a sensitivity of $\sim 0.1 \mathrm{fmol}$ (corresponding to a concentration of $10^{-5} \mathrm{M}$ ). In other words, the smaller R6G concentration is not detected with Ag@PVP10000-based sensor. The relation of the normalized areas under the main vibrational bands of the R6G obtained in this experiments demonstrates that both capped Ag(H) and Ag@PVP29000 nanoparticles present equivalent amplification factor within experimental variations up to $10^{-7} \mathrm{M}$ (see the detail of the spectra shown in Figure 5). In the first case, and after the aggregation, the high degree of coverage of the silicon films together with the proximity of plasmon absorption of the nanoparticle aggregates to the excitation radiation work in the favor of a great enhancement of the Raman signal. In the second case, since the degree of coverage is deficient with respect to the $\operatorname{Ag}(\mathrm{H})$ surfaces and the hot spot size is similar to those of the $\operatorname{Ag}(\mathrm{H})$ films, only the electronic properties are of advantage with respect to these sensors. The longitudinal plasmon absorption of the nanotriangles is more intense than the absorption of the broad distribution of $\operatorname{Ag}(\mathrm{H})$ aggregates and is also closer to the laser wavelength. Consequently, Ag@PVP29000 would be benefited for an improvement of the surface coverage homogeneity. Current work is being devoted in such direction with promising results employing functionalization with $\beta$-cyclodextrin as analyte captor.

Finally, reversibility of these thiol-immobilized silver nanoparticle-based nanostructures is probed by means of washing them for $1 \mathrm{~h}$ with Milli-Q water after incubating with R6G $10^{-7} \mathrm{M}$. This reversibility has not been measured in the PVP10000-based sensor, since this sensitivity is not achieved in this case. Results for Ag@PVP29000 films are presented in Figure 7 as an example demonstrating this 


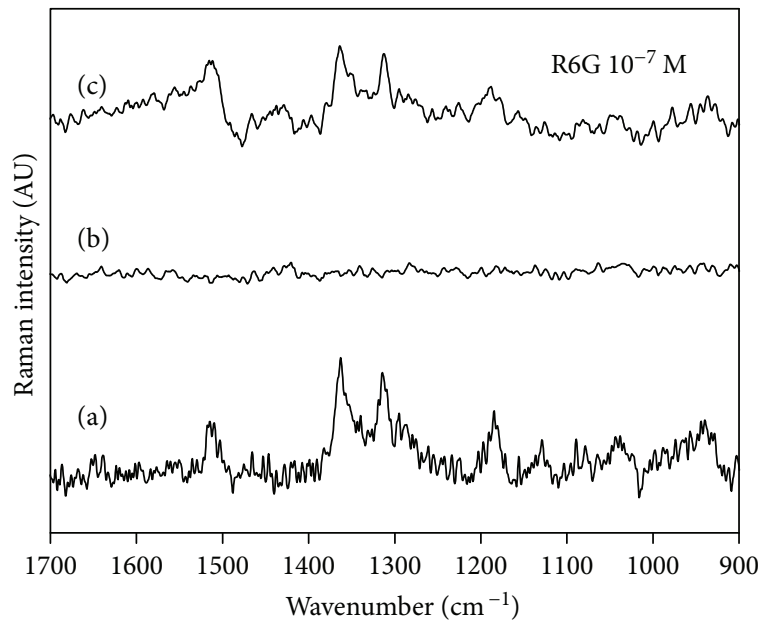

Figure 7: Surface-enhanced Raman scattering spectra recorded on the Ag@polyvinylpyrrolidone-29000-based sensor after (a) incubating it in rhodamine $6 \mathrm{G} 10^{-7} \mathrm{M}$ for $1 \mathrm{~h}$, (b) thorough washing in Milli-Q water for $1 \mathrm{~h}$, and (c) reincubating it in rhodamine $6 \mathrm{G} 10^{-7} \mathrm{M}$. Similar results are obtained with the films composed of attached $\mathrm{Ag}(\mathrm{H})$ nanoparticles.

feature that makes them candidates for reusable chemical sensors. Figure 7(a), where the spectrum of the film incubated in $\mathrm{R} 6 \mathrm{G} 10^{-7} \mathrm{M}$ for $1 \mathrm{~h}$ is exhibited, shows clearly the R6G bands pattern. Spectrum B was measured after washing the film for $1 \mathrm{~h}$ in Milli-Q water, showing no SERS signals from R6G. Finally, spectrum C, recorded after reincubating in the R6G $10^{-7} \mathrm{M}$ solution, shows clearly a shape that is rather similar to that in spectrum $\mathrm{A}$.

\section{Conclusions}

Three substrates for organic compound detection based on bare and PVP-capped metal nanoparticles have been developed. These metal nanoparticles come from three different syntheses and have been characterized with a plethora of techniques. They are strongly attached to a silicon substrate, and hence, they can be incubated without danger of degradation of SERS active spots. Aggregates formed by core Ag nanoparticles are of micrometric size while capped ones hardly form a $10 \mu \mathrm{m}$ aggregate due to the steric effect caused by the functional group size. Therefore, the coverage of the films will decrease with a bulky coating. The plasmon properties of the nanoparticles are, in its hand, controlled by their shape and are the leading order term within the variety of effects implicated in the Raman process. This twofold effect (electronic and area factors) controls SERS signal and can be finely tuned by a proper choice of coating and synthesis parameters. With an appropriate excitation light, reversibility of the adsorption-desorption process of the organic compound makes these modified films perfect candidates for being used as chemical sensor based on the SERS effect. These results put the pave for a new methodology on the way to reusable molecular sensors based on Raman spectroscopy. Further insights into the hydrophobic/hydrophilic effect of the substrates as long as new functionalization aims to increase the analyte capture are currently being explored with exciting perspectives.

\section{Conflicts of Interest}

The authors declare that they have no conflicts of interest.

\section{Acknowledgments}

C. Caro and F. Gámez acknowledge funding from Junta de Andalucía through projects P07-FQM-02595 and P07FQM-02600 for their postdoctoral fellowship, respectively. This study is in memory of Jose Antonio Mejías Romero.

\section{Supplementary Materials}

The supplementary material section contains the detailed characterization of the treated substrate characterization. Figure S1 shows the FTIR spectrum of the $\mathrm{Si}(100)$ chip after treatment with piranha solution for $1 \mathrm{~h}$. Then the sample has been rinsed in Milli-Q water and dried with nitrogen. The spectrum of liquid water, recorded in a 10 reflection ZnSe ATR, is also shown for comparison as discussed in the text. Adapted from Caro et al. [50]. A brief explanation of the experiments carried out to rule out non-SERS contributions to the observed signal is also included. (Supplementary Materials)

\section{References}

[1] X. J. Huang and Y. K. Choi, "Chemical sensors based on nanostructured materials," Sensors and Actuators B: Chemical, vol. 122, no. 2, pp. 659-671, 2007.

[2] K. Cao, X. Jiang, S. Yan, L. Zhang, and W. Wu, "Phenylboronic acid modified silver nanoparticles for colorimetric dynamic analysis of glucose," Biosensors and Bioelectronics, vol. 52, pp. 188-195, 2014.

[3] G. De Micheli, "Nanoelectronics: challenges and opportunities," in Integrated Circuit and System Design: Power and Timing Modeling, Optimization and Simulation, Springer, Berlin, Heidelberg, 2006.

[4] B. Tian and C. M. Lieber, "Synthetic nanoelectronic probes for biological cells and tissues," Annual Review of Analytical Chemistry, vol. 6, no. 1, pp. 31-51, 2013.

[5] L. Novotny and B. Hecht, Eds., Principles of Nano-Optics, Cambridge University Press, Cambridge, UK, 2006.

[6] V. M. Shalaev and S. Kawata, Nanophotonics with Surface Plasmons, Elsevier, Amsterdam, The Netherlands, 2007.

[7] D. L. Feldheim and C. A. Foss, Metal Nanoparticles. Synthesis, Characterization and Applications, Marcel Dekker, Inc., New York, NY, USA, 2002.

[8] A. Inberg, P. Livshits, Z. Zalevsky, and Y. Shacham-Diamand, "Electroless deposition of silver thin films on gold nanoparticles catalyst for micro and nanoelectronics applications," Microelectronic Engineering, vol. 98, pp. 570-573, 2012.

[9] A. W. Castleman Jr. and S. N. Khanna, "Clusters, superatoms, and building blocks of new materials," Journal of Physical Chemistry C, vol. 113, no. 7, pp. 2664-2675, 2009.

[10] S. K. Ghosh, A. Pal, S. Nath, S. Kundu, S. Panigrahi, and T. Pal, "Dimerization of eosin on nanostructured gold surfaces: size regime dependence of the small metallic particles," Chemical Physics Letters, vol. 412, no. 1-3, pp. 5-11, 2005. 
[11] R. Kubo, "Electronic properties of metallic fine particles. I," Journal of the Physical Society of Japan, vol. 17, no. 6, pp. 975-986, 1962.

[12] L. Xie, X. Huang, B. W. Li, C. Zhi, T. Tanaka, and P. Jiang, "Core-satellite Ag@ $\mathrm{BaTiO}_{3}$ nanoassemblies for fabrication of polymer nanocomposites with high discharged energy density, high breakdown strength and low dielectric loss," Physical Chemistry Chemical Physics, vol. 15, no. 40, pp. 17560$17569,2013$.

[13] J. D. Jackson, Classical Electrodynamics, vol. 1975, Wiley, New York, NY, USA, 1975.

[14] M. Brack, "The physics of simple metal clusters: self-consistent jellium model and semiclassical approaches," Reviews of Modern Physics, vol. 65, no. 3, pp. 677-732, 1993.

[15] V. López-Puente, S. Abalde-Cela, P. C. Angelomé, R. A. Alvarez-Puebla, and L. M. Liz-Marzán, "Plasmonic mesoporous composites as molecular sieves for SERS detection," The Journal of Physical Chemistry Letters, vol. 4, no. 16, pp. 2715-2720, 2013.

[16] R. P. Devaty and A. J. Sievers, "Possibility of observing quantum size effects in the electromagnetic absorption spectrum of small metal particles," Physical Review B, vol. 32, no. 4, pp. 1951-1954, 1985.

[17] P. B. Johnson and R. W. Christy, "Optical constants of the noble metals," Physics Review B, vol. 6, no. 12, pp. 43704379, 1972.

[18] L. S. Jung, C. T. Campbell, T. M. Chinowsky, M. N. Mar, and S. S. Yee, "Quantitative interpretation of the response of surface plasmon resonance sensors to adsorbed films," Langmuir, vol. 14, no. 19, pp. 5636-5648, 1998.

[19] K. Kneipp, M. Moskovits, and H. Kneipp, Eds., SurfaceEnhanced Raman Scattering: Physics and Applications, Springer-Verlag, Berlin Heidelberg, 2006.

[20] P. Quaresma, I. Osório, G. Dória et al., "Star-shaped magnetite@gold nanoparticles for protein magnetic separation and SERS detection," RSC Advances, vol. 4, no. 8, pp. 3690-3698, 2014.

[21] S. Schlücker, "Surface-enhanced Raman spectroscopy: concepts and chemical applications," Angewandte Chemie International Edition, vol. 53, no. 19, pp. 4756-4795, 2014.

[22] A. Araújo, C. Caro, M. J. Mendes et al., "Highly efficient nanoplasmonic SERS on cardboard packaging substrates," Nanotechnology, vol. 25, no. 41, article 415202, 2014.

[23] M. Cueto, M. Piedrahita, C. Caro et al., "Platinum nanoparticles as photoactive substrates for mass spectrometry and spectroscopy sensors," Journal of Physical Chemistry C, vol. 118, no. 21, pp. 11432-11439, 2014.

[24] K. Kneipp and H. Kneipp, "Surface enhanced Raman scattering-a tool for ultrasensitive trace analysis," Canadian Journal of Analytical Sciences and Spectroscopy, vol. 48, pp. 125-131, 2003.

[25] K. Kneipp, H. Kneipp, I. Itzkan, R. R. Dasari, and M. S. Feld, "Surface-enhanced Raman scattering: a new tool for biomedical spectroscopy," Current Science, vol. 77, pp. 915-924, 1999.

[26] R. A. Alvarez-Puebla and L. M. Liz-Marzán, "SERS detection of small inorganic molecules and ions," Angewandte Chemie International Edition, vol. 51, no. 45, pp. 11214-11223, 2012.

[27] K. Kneipp, H. Kneipp, I. Itzkan, R. R. Dasari, and M. S. Feld, "Ultrasensitive chemical analysis by Raman spectroscopy," Chemical Reviews, vol. 99, no. 10, pp. 2957-2976, 1999.
[28] L. Chen, J. M. Chabu, R. Jin, and J. Xiao, "Single goldnanoparticles-decorated silver/carbon nanowires as substrates for surface-enhanced Raman scattering detection," RSC Advances, vol. 3, no. 48, pp. 26102-26109, 2013.

[29] K. Kneipp, H. Kneipp, I. Itzkan, R. R. Dasari, and M. S. Feld, "Surface-enhanced Raman scattering and biophysics," Journal of Physics. Condensed Matter, vol. 14, no. 18, pp. R597-R624, 2002.

[30] D. Pan and D. L. Phillips, "FT-surface-enhanced Raman scattering of $2,2^{\prime}$-cyanine adsorbed on silver with AFM characterization of silver films," Chemical Physics Letters, vol. 275, no. 3-4, pp. 227-233, 1997.

[31] J. R. Lombardi and R. L. Birke, "A unified approach to surfaceenhanced Raman spectroscopy," Journal of Physical Chemistry C, vol. 112, no. 14, pp. 5605-5617, 2008.

[32] Z. Zhang and T. Imae, "Study of surface-enhanced infrared spectroscopy: 1. Dependence of the enhancement on thickness of metal island films and structure of chemisorbed molecules," Journal of Colloid and Interface Science, vol. 233, no. 1, pp. 99106, 2001

[33] L. K. Ausman, S. Li, and G. C. Schatz, "Structural effects in the electromagnetic enhancement mechanism of surfaceenhanced Raman scattering: dipole reradiation and rectangular symmetry effects for nanoparticle arrays," Journal of Physical Chemistry C, vol. 116, no. 33, pp. 17318-17327, 2012.

[34] H. Guo, W. Xu, J. Zhou, S. Xu, and J. R. Lombardi, "Highly efficient construction of oriented sandwich structures for surface-enhanced Raman scattering," Nanotechnology, vol. 24, no. 4, article 045608, 2013.

[35] C. Caro, M. J. Sayagues, V. Franco, A. Conde, P. Zaderenko, and F. Gámez, "A hybrid silver-magnetite detector based on surface enhanced Raman scattering for differentiating organic compounds," Sensors and Actuators B: Chemical, vol. 228, pp. 124-133, 2016.

[36] P. Johansson, H. Xu, and M. Käll, "Surface-enhanced Raman scattering and fluorescence near metal nanoparticles," Physical Review B, vol. 72, no. 3, article 035427, 2005.

[37] W. Yue, Z. Wang, X. Wang et al., "Fabrication of metallic nanostructures of sub-20 nm with an optimized process of E-beam lithography and lift-off," Journal of Nanoscience and Nanotechnology, vol. 12, no. 1, pp. 696-699, 2012.

[38] R. Eason, Pulsed Laser Deposition of Thin Films: Applications-Led Growth of Functional Materials, R. Eason, Ed., Wiley-Interscience, University of Michigan, 2007.

[39] L. A. Dick, A. D. McFarland, C. L. Haynes, and R. P. Van Duyne, "Metal film over nanosphere (MFON) electrodes for surface-enhanced Raman spectroscopy (SERS): improvements in surface nanostructure stability and suppression of irreversible loss," The Journal of Physical Chemistry. B, vol. 106, no. 4, pp. 853-860, 2002.

[40] M. Litorja, C. L. Haynes, A. J. Haes, T. R. Jensen, and R. P. Van Duyne, "Surface-enhanced Raman scattering detected temperature programmed desorption: optical properties, nanostructure, and stability of silver film over $\mathrm{SiO}_{2}$ nanosphere surfaces," The Journal of Physical Chemistry. B, vol. 105, no. 29, pp. 6907-6915, 2001.

[41] G. Sauer, G. Brehm, and S. Schneider, "Preparation of SERS-active gold film electrodes via electrocrystallization: their characterization and application with NIR excitation," Journal of Raman Spectroscopy, vol. 35, no. 7, pp. 568576, 2004. 
[42] J. H. Dickerson and A. R. Boccaccini, Electrophoretic Deposition of Nanomaterials, Springer, New York, NY, USA, 2012.

[43] K. X. Xu, M. H. Guo, Y. P. Huang, X. D. Li, and J. J. Sun, "Rapid and sensitive detection of malachite green in aquaculture water by electrochemical preconcentration and surfaceenhanced Raman scattering," Talanta, vol. 180, pp. 383-388, 2018.

[44] B. S. Lee, P. C. Lin, D. Z. Lin, and T. J. Yen, "Rapid biochemical mixture screening by three-dimensional patterned multifunctional substrate with ultra-thin layer chromatography (UTLC) and surface enhanced Raman scattering (SERS)," Scientific Reports, vol. 8, no. 1, p. 516, 2018.

[45] J. Hu, B. Zhao, W. Xu, Y. Fan, B. Li, and Y. Ozaki, "Simple method for preparing controllably aggregated silver particle films used as surface-enhanced Raman scattering active substrates," Langmuir, vol. 18, no. 18, pp. 6839$6844,2002$.

[46] P. Leyton, S. Sanchez-Cortes, J. V. Garcia-Ramos et al., "Selective molecular recognition of polycyclic aromatic hydrocarbons (PAHs) on calix[4]arene-functionalized $\mathrm{Ag}$ nanoparticles by surface-enhanced Raman scattering," The Journal of Physical Chemistry. B, vol. 108, no. 45, pp. 17484-17490, 2004.

[47] L. Shu, J. Zhou, X. Yuan et al., "Highly sensitive immunoassay based on SERS using nano-Au immune probes and a nano-Ag immune substrate," Talanta, vol. 123, pp. 161$168,2014$.

[48] M. V. Canamares, P. Sevilla, S. Sanchez-Cortes, and J. V. Garcia-Ramos, "Surface-enhanced Raman scattering study of the interaction of red dye alizarin with ovalbumin," Biopolymers, vol. 82, no. 4, pp. 405-409, 2006.

[49] Y. Wang, H. Chen, S. Dong, and E. Wang, "Fabrication and characterization of SERS-active silver clusters on glassy carbon," Journal of Raman Spectroscopy, vol. 38, no. 5, pp. 515521, 2007.

[50] C. Caro, C. López-Cartes, P. Zaderenko, and J. A. Mejías, "Thiol-immobilized silver nanoparticle aggregate films for surface enhanced Raman scattering," Journal of Raman Spectroscopy, vol. 39, no. 9, pp. 1162-1169, 2008.

[51] N. Leopold and B. Lendl, "A new method for fast preparation of highly surface-enhanced Raman scattering (SERS) active silver colloids at room temperature by reduction of silver nitrate with hydroxylamine hydrochloride," The Journal of Physical Chemistry. B, vol. 107, no. 24, pp. 57235727, 2003.

[52] I. Washio, Y. Xiong, Y. Yin, and Y. Xia, "Reduction by the end groups of poly(vinyl pyrrolidone): a new and versatile route to the kinetically controlled synthesis of Ag triangular nanoplates," Advanced Materials, vol. 18, no. 13, pp. 1745-1749, 2006.

[53] G. S. S. Saini, S. Kaur, S. K. Tripathi, C. G. Mahajan, H. H. Thanga, and A. L. Verma, "Spectroscopic studies of rhodamine 6G dispersed in polymethylcyanoacrylate," Spectrochimica Acta Part A: Molecular and Biomolecular Spectroscopy, vol. 61, no. 4, pp. 653-658, 2005.

[54] L. Guerrini, J. V. Garcia-Ramos, C. Domingo, and S. SanchezCortes, "Nanosensors based on viologen functionalized silver nanoparticles: few molecules surface-enhanced Raman spectroscopy detection of polycyclic aromatic hydrocarbons in interparticle hot spots," Analytical Chemistry, vol. 81, no. 4, pp. 1418-1425, 2009.
[55] C. Andreou, M. R. Hoonejani, M. R. Barmi, M. Moskovits, and C. D. Meinhart, "Rapid detection of drugs of abuse in saliva using surface enhanced Raman spectroscopy and microfluidics," ACS Nano, vol. 7, no. 8, pp. 7157-7164, 2013.

[56] E. C. Le Ru, E. Blackie, M. Meyer, and P. G. Etchegoin, "Surface enhanced Raman scattering enhancement factors: a comprehensive study," Journal of Physical Chemistry C, vol. 111, no. 37, pp. 13794-13803, 2007. 

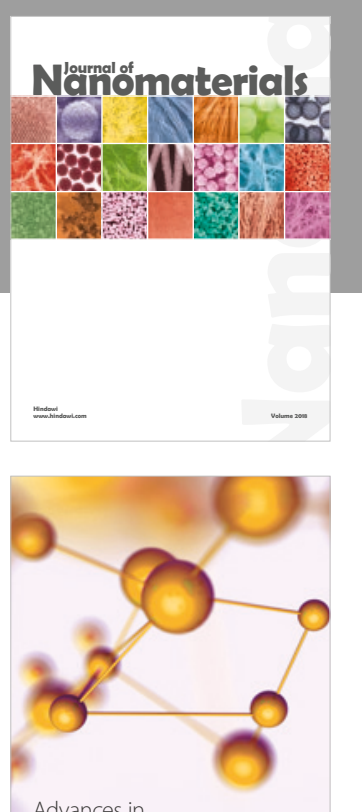

Physical Chemistry
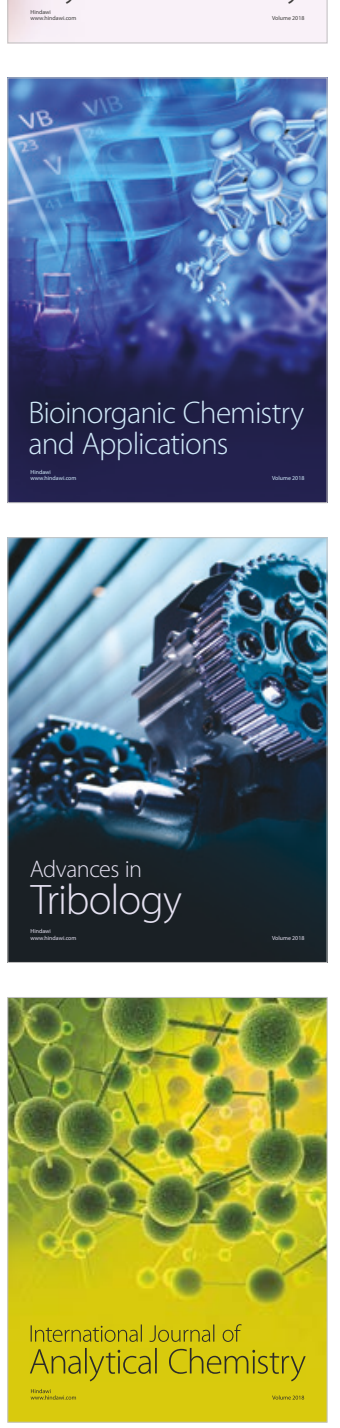

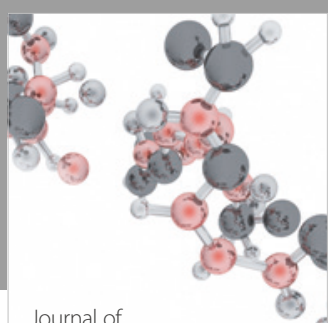

Analytical Methods

in Chemistry

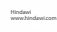

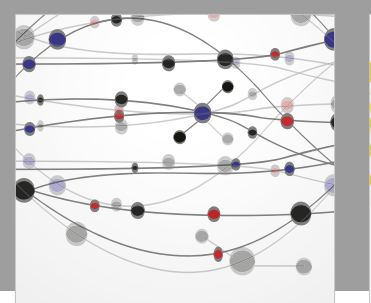

The Scientific World Journal

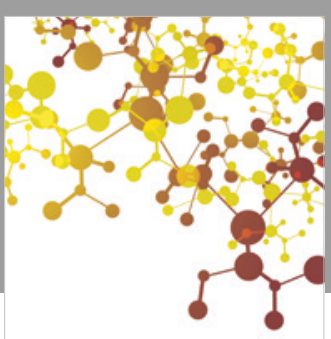

Journal of

Applied Chemistry
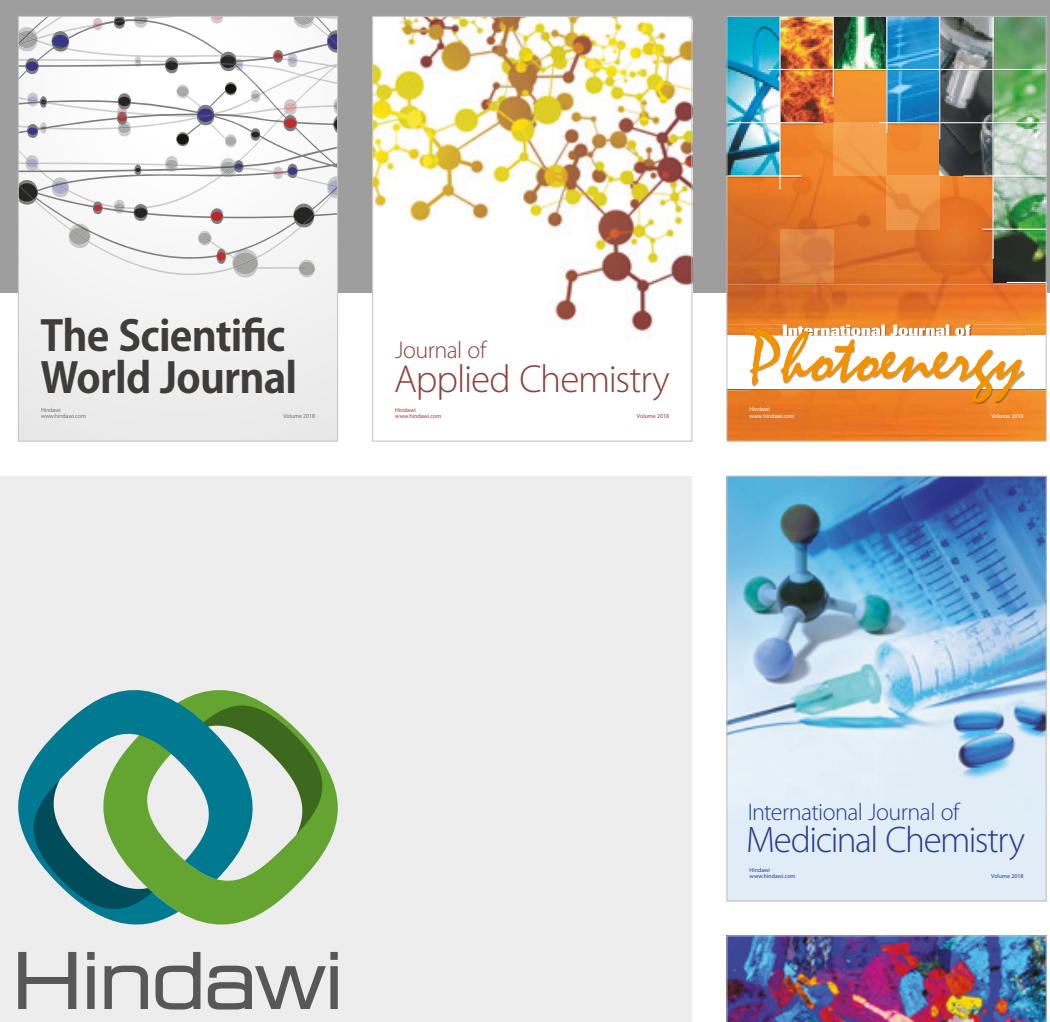

Submit your manuscripts at

www.hindawi.com
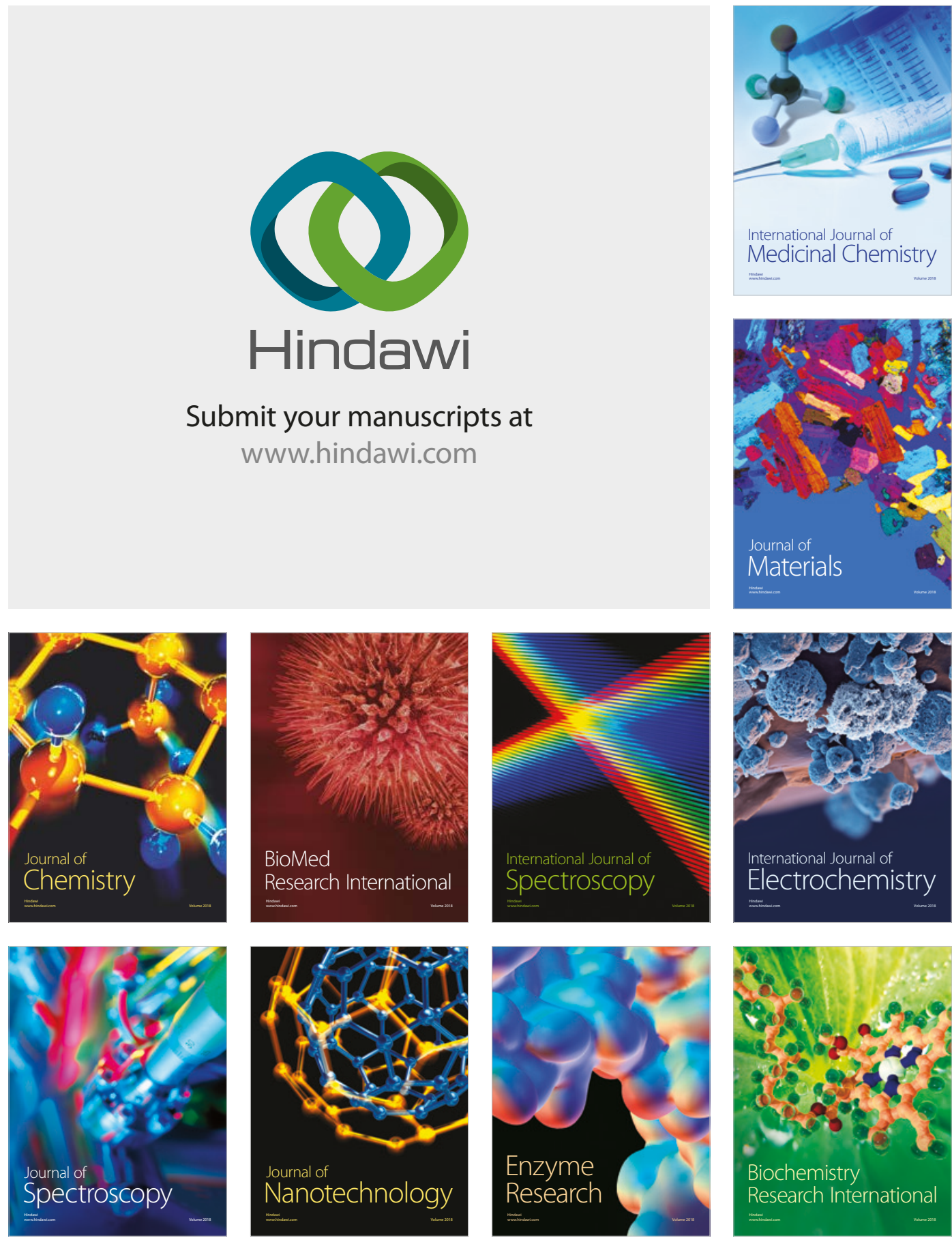
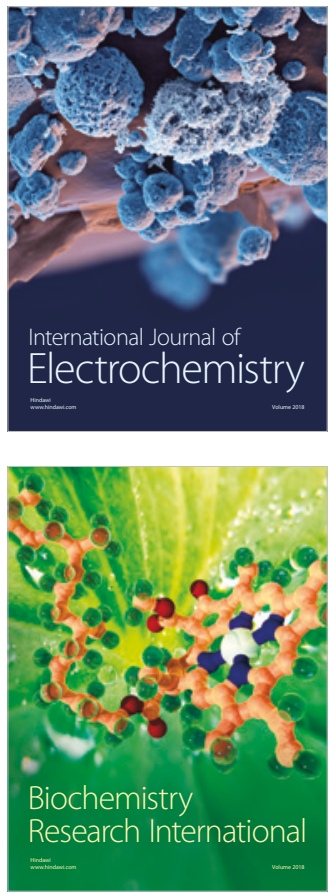